\title{
ON THE DETOUR AND VERTEX DETOUR HULL NUMBERS OF A GRAPH
}

\author{
A.P. Santhakumaran ${ }^{1}$ And S.V. Ullas Chandran ${ }^{2}$ \\ ${ }^{1}$ Department of Mathematics Hindustan University Hindustan Institute of \\ Technology and Science, Padur, Chennai - 603 103, India \\ apskumar1953@yahoo.co.in \\ ${ }^{2}$ Department of Mathematics, Mahatma Gandhi College, Kesavdasapuram, \\ Pattom P.O., Thiruvanathapuram - 695 004, India \\ svuc.math@gmail.com
}

\begin{abstract}
For vertices $x$ and $y$ in a connected graph $G$, the detour distance $D(x, y)$ is the length of a longest $x-y$ path in $G$. An $x-y$ path of length $D(x, y)$ is an $x-y$ detour. The closed detour interval $I_{D}[x, y]$ consists of $x, y$, and all vertices lying on some $x-y$ detour of $G$; while for $S \subseteq V(G), I_{D}[S]=\bigcup_{x, y \in S} I_{D}[x, y]$. A set $S$ of vertices is a detour convex set if $I_{D}[S]=S$. The detour convex hull $[S]_{D}$ is the smallest detour convex set containing $S$. The detour hull number $d h(G)$ is the minimum cardinality among subsets $S$ of $V(G)$ with $[S]_{D}=V(G)$. Let $x$ be any vertex in a connected graph $G$. For a vertex $y$ in $G$, denote by $I_{G}[y]^{x}$, the set of all vertices distinct from $x$ that lie on some $x-y$ detour of $G$; while for $S \subseteq V(G), I_{D}[S]^{x}=\bigcup_{y \in S} I_{D}[y]^{x}$. For $x \notin S, S$ is an $x$-detour set of $G$ if $I_{D}[S]^{x}=V(G)-\{x\}$ and an $x$-detour set of minimum cardinality is the $x$-detour number $d_{x}(G)$ of $G$. For $x \notin S, S$ is an $x$-detour convex set if $I_{D}[S]^{x}=S$. The $x$-detour convex hull of $S,[S]_{D}^{x}$ is the smallest $x$-detour convex set containing $S$. The $x$-detour hull number $d h_{x}(G)$ is the minimum cardinality among the subsets $S$ of $V(G)-\{x\}$ with $[S]_{D}^{x}=V(G)-\{x\}$. In this paper, we investigate how the detour hull number and the vertex detour hull number of a connected graph are affected by adding a pendant edge.
\end{abstract}

Key words and Phrases:d Detour, detour number, detour hull number, $x$-detour number, $x$-detour hull number.

2000 Mathematics Subject Classification: 05C12

Received: 27-08-2013, revised: 21-01-2014, accepted: 21-01-2014. 


\begin{abstract}
Abstrak. Misalkan $x$ dan $y$ berada di graf terhubung $G$, jarak detour $D(x, y)$ adalah panjang dari lintasan $x-y$ yang terpanjang di $G$. Lintasan $x-y$ dengan panjang $D(x, y)$ adalah suatu detour $x-y$. Interval detour tertutup $I_{D}[x, y]$ memuat $x, y$ dan semua titik yang berada dalam suatu detour $x-y$ dari $G$; sedangkan untuk $S \subseteq V(G), I_{D}[S]=\bigcup_{x, y \in S} I_{D}[x, y]$. Himpunan titik $S$ adalah suatu himpunan konveks detour jika $I_{D}[S]=S$. Konveks hull detour $[S]_{D}$ adalah himpunan konveks detour terkecil yang memuat $S$. Bilangan hull detour $d h(G)$ adalah kardinalitas minimum diantara sub-subhimpunan $S$ dari $V(G)$ dengan $[S]_{D}=V(G)$. Misalkan $x$ adalah suatu titik di graf terhubung $G$. Untuk suatu titik $y$ di $G$, dinotasikan dengan $I_{G}[y]^{x}$, himpunan dari semua titik berbeda dari $x$ yang terletak pada suatu detour $x-y$ dari $G$; sedangkan untuk $S \subseteq V(G), I_{D}[S]^{x}=\bigcup_{y \in S} I_{D}[y]^{x}$. Untuk $x \notin S, S$ adalah suatu himpuan detour- $x$ dari $G$ jika $I_{D}[S]^{x}=V(G)-\{x\}$ dan suatu himpuan detour- $x$ dengan kardinalitas minimum adalah bilangan detour- $x d_{x}(G)$ dari $G$. Untuk $x \notin S, S$ adalh suatu himpunan detour- $x$ konveks jika $I_{D}[S]^{x}=$ $S$. Konveks hull detour- $x$ dari $S,[S]_{D}^{x}$ adalah himpunan konveks detour- $x$ yang memuat $S$. Bilangan hull detour- $x d h_{x}(G)$ adalah kardinalitas minimum diantara sub-subhimpunan $S$ dari $V(G)-\{x\}$ dengan $[S]_{D}^{x}=V(G)-\{x\}$. Pada paper ini, kami memeriksa pengaruh penambahan sisi anting dari suatu graf terhubung terhadap bilangan hull detour dan bilangan hull detour titik.
\end{abstract}

Kata kunci: Detour, bilangan detour, bilangan hull detour, bilangan detour- $x$, bilangan hull detour- $x$.

\title{
1. INTRODUCTION
}

By a graph $G=(V, E)$, we mean a finite undirected graph without loops or multiple edges. The order and size of $G$ are denoted by $n$ and $m$ respectively. For basic definitions and terminologies, we refer to $[1,6]$. For vertices $x$ and $y$ in a nontrivial connected graph $G$, the detour distance $D(x, y)$ is the length of a longest $x-y$ path in $G$. An $x-y$ path of length $D(x, y)$ is an $x-y$ detour. It is known that the detour distance is a metric on the vertex set $V(G)$. The detour eccentricity of a vertex $u$ is $e_{D}(u)=\max \{D(u, v): v \in V(G)\}$. The detour radius, $\operatorname{rad}_{D}(G)$ of $G$ is the minimum detour eccentricity among the vertices of $G$, while the detour diameter, $\operatorname{diam}_{D}(G)$ of $G$ is the maximum detour eccentricity among the vertices of $G$. The detour distance and the detour center of a graph were studied in [2]. The closed detour interval $I_{D}[x, y]$ consists of $x, y$, and all vertices lying on some $x-y$ detour of $G$; while for $S \subseteq V(G), I_{D}[S]=\bigcup_{x, y \in S} I_{D}[x, y] ; S$ is a detour set if $I_{D}[S]=V(G)$ and a detour set of minimum cardinality is the detour number $d n(G)$ of $G$. Any detour set of cardinality $d n(G)$ is the minimum detour set or $d n$-set of $G$. A vertex $x$ in $G$ is a detour extreme vertex if it is an initial or terminal vertex of any detour containing $x$. The detour number of a graph was introduced in [3] and further studied in $[4,8]$. These concepts have interesting applications in Channel Assignment Problem in radio technologies $[5,7]$. 
A set $S$ of vertices of a graph $G$ is a detour convex set if $I_{D}[S]=S$. The detour convex hull $[S]_{D}$ of $S$ is the smallest detour convex set containing $S$. The detour convex hull of $S$ can also be formed from the sequence $\left\{I_{D}^{k}[S], k \geq 0\right\}$, where $I_{D}^{0}[S]=S, I_{D}^{1}[S]=I_{D}[S]$ and $I_{D}^{k}=I_{D}\left[I_{D}^{k-1}[S]\right]$. From some term on, this sequence must be constant. Let $p$ be the smallest number such that $I_{D}^{p}[S]=I_{D}^{p+1}[S]$. Then $I_{D}^{p}[S]$ is the detour convex hull $[S]_{D}$ and we call $p$ as the detour iteration number $\operatorname{din}(S)$ of $S$. A set $S$ of vertices of $G$ is a detour hull set if $[S]_{D}=V(G)$ and a detour hull set of minimum cardinality is the detour hull number $d h(G)$. The detour hull number of a graph was introduced and studied in [11].

For the graph $G$ given in Figure 1, and $S=\left\{v_{1}, v_{6}\right\}, I_{D}[S]=V-\left\{v_{7}\right\}$ and $I_{D}^{2}[S]=V$. Thus $S$ is a minimum detour hull set of $G$ and so $d_{h}(G)=2$. Since $S$ is not a detour set and $S \cup\left\{v_{7}\right\}$ is a detour set of $G$, it follows from Theorem 1.2 that $d n(G)=3$. Hence the detour number and detour hull number of a graph are different. Note that the sets $S_{1}=\left\{v_{1}, v_{2}\right\}$ and $S_{2}=\left\{v_{2}, v_{3}, v_{4}, v_{5}, v_{7}\right\}$ are detour convex sets in $G$. Let $x$ be any vertex of $G$. For a vertex $y$ in $G, I_{G}[y]^{x}$ denotes

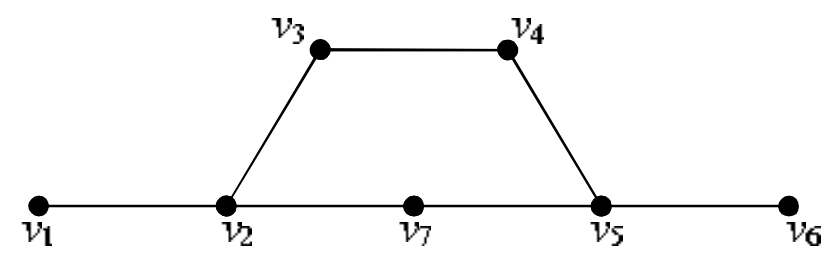

Figure 1. Graph $G$ with $d_{h}(G)=2$ and $d n(G)=3$

the set of all vertices distinct from $x$ that lie on some $x-y$ detour of $G$; while for $S \subseteq V(G), I_{D}[S]^{x}=\bigcup_{y \in S} I_{D}[y]^{x}$. It is clear that $I_{D}[x]^{x}=\phi$. For $x \notin S, S$ is an $x$-detour set if $I_{D}[S]^{x}=V(G)-\{x\}$ and an $x$-detour set of minimum cardinality is the $x$-detour number $d_{x}(G)$ of $G$. Any $x$-detour set of cardinality $d_{x}(G)$ is the minimum $x$-detour set or $d_{x}$-set of $G$. The vertex detour number of a graph was introduced and studied in [9].

Let $G$ be a connected graph and $x$ a vertex in $G$. Let $S$ be a set of vertices in $G$ such that $x \notin S$. Then $S$ is an $x$-detour convex set if $I_{D}[S]^{x}=S$. The $x$-detour convex hull of $S,[S]_{D}^{x}$ is the smallest $x$-detour convex set containing $S$. The $x$-detour convex set can also formed from the sequence $\left\{I_{D}^{k}[S]^{x}, k \geq 0\right\}$, where $I_{D}^{0}[S]^{x}=S, I_{D}^{1}[S]^{x}=I_{D}[S]^{x}$ and $I_{D}^{k}[S]^{x}=I_{D}\left[I_{D}^{k-1}[S]^{x}\right]^{x}$. From some term on, this sequence must be constant. Let $p_{x}$ be the smallest number such that $I_{D}^{p_{x}}[S]^{x}=$ $I_{D}^{p_{x}+1}[S]^{x}$. Then $I_{D}^{p_{x}}[S]^{x}$ is the $x$-detour convex hull $[S]_{D}^{x}$ of $S$ and we call $p_{x}$ as the $x$-detour iteration number $\operatorname{din}_{x}(S)$ of $S$. The set $S$ is an $x$-detour hull set if $[S]_{D}^{x}=V(G)-\{x\}$ and an $x$-detour hull set of minimum cardinality is the $x$ detour hull number $d h_{x}(G)$ of $G$. Any $x$-detour hull set of cardinality $d h_{x}(G)$ is the minimum $x$-detour hull set or $d_{x}$-hull set of $G$.

For the graph $G$ in Figure 2, the minimum vertex detour hull numbers and vertex detour numbers are given in Table 1 . Table 1 shows that, for a vertex $x$, the $x$-detour number and the $x$-detour hull number of a graph are different. 


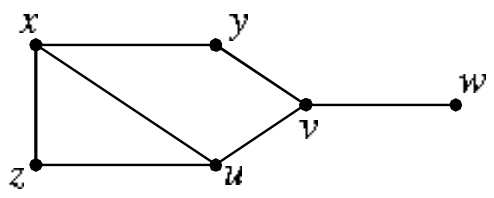

Figure 2. $G$

Table 1. $x$-detour numbers and $x$-detour hull numbers of $G$ in Figure 2

\begin{tabular}{|c|c|c|c|c|}
\hline Vertex & $\begin{array}{l}\text { Minimum verter } \\
\text { detour sets }\end{array}$ & $\begin{array}{l}\text { Minimum vertex detour } \\
\text { hull sets }\end{array}$ & $\begin{array}{l}\text { Vertex } \\
\text { detour } \\
\text { number }\end{array}$ & $\begin{array}{c}\text { Vertex } \\
\text { detour hull } \\
\text { number }\end{array}$ \\
\hline$x$ & $\{y, w\},\{z, w\},\{u, w\}$ & $\{w\}$ & 2 & 1 \\
\hline $\mathrm{y}$ & $\{w\}$ & $\{w\}$ & 1 & 1 \\
\hline$z$ & $\{w\}$ & $\{w\}$ & 1 & 1 \\
\hline$u$ & $\{w\}$ & $\{w\}$ & 1 & 1 \\
\hline$v$ & $\{y, w\},\{z, w\},\{u, w\}$ & $\{x, w\},\{y, w\},\{z, w\},\{u, w\}$ & 2 & 2 \\
\hline$w$ & $\{y\},\{z\},\{u\}$ & $\{x\},\{y\},\{z\},\{u\}$ & 1 & 1 \\
\hline
\end{tabular}

It is clear that every minimum $x$-detour hull set of a connected graph $G$ of order $n$ contains at least one vertex and at most $n-1$ vertices. Also, since every $x$-detour set is a $x$-detour hull set, we have the following proposition. Throughout this paper $G$ denotes a connected graph with at least two vertices. The following theorems will be used in the sequel.

Theorem 1.1. [11] Let $G$ be a connected graph. Then

(i) Each detour extreme vertex of $G$ belongs to every detour hull set of $G$.

(ii) No cut vertex of $G$ belongs to any minimum detour hull set of $G$.

Theorem 1.2. [9] Each end vertex of $G$ other than $x$ (whether $x$ is an end vertex or not) belongs to every minimum $x$-detour set of $G$.

Theorem 1.3. [10] Let $x$ be a vertex of a connected graph $G$. Let $S$ be any $x$-detour hull set of $G$. Then

(i) Each $x$-detour extreme vertex of $G$ belongs to $S$.

(ii) If $v$ is a cut vertex of $G$ and $C$ a component of $G-v$ such that $x \notin V(C)$, then $S \cap V(C) \neq \emptyset$.

(iii) No cut-vertex of $G$ belongs to any minimum $x$-detour hull set of $G$.

Theorem 1.4. [10] For any vertex $x$ in a connected graph $G$ of order $n, d h_{x}(G) \leq$ $n-e_{D}(x)$.

2. Graphs of Order $n$ With Vertex Detour Hull Number $n-1, n-2$ AND $n-3$

Theorem 2.1. Let $G$ be a connected graph of order $n \geq 2$. Then $d h_{x}(G)=n-1$ for every vertex $x$ in $G$ if and only if $G=K_{2}$. 
Proof. Suppose that $G=K_{2}$. Then $d h_{x}(G)=1=n-1$. The converse follows from Theorem 1.4.

Theorem 2.2. Let $G$ be a connected graph of order $n \geq 3$. Then $d h_{x}(G)=n-2$ for every vertex $x$ in $G$ if and only if $G=K_{3}$.

Proof. Suppose that $G=K_{3}$. Then it is clear that $d h_{x}(G)=1=n-2$ for every vertex $x$ in $G$. Conversely, suppose that $d h_{x}(G)=n-2$ for every vertex $x$ in $G$. Then by Theorem 1.4, $e_{D}(x) \leq 2$ for every vertex $x$ in $G$. It follows from Theorem 2.1 that $e_{D}(x) \neq 1$ for every vertex $x$ in $G$. Thus $e_{D}(x)=2$ for every vertex $x$ in $G$; or the vertex set can be partitioned into $V_{1}$ and $V_{2}$ such that $e_{D}(x)=1$ for $x \in V_{1}$ and $e_{D}(x)=2$ for $x \in V_{2}$. Thus either $\operatorname{rad}_{D}(G)=\operatorname{diam}_{D}(G)=2$; or we have $\operatorname{rad}_{D}(G)=1$ and $\operatorname{diam}_{D}(G)=2$. This implies that either $G=K_{3}$ or $G=K_{1, n-1}$. If $G=K_{1, n-1}$, then by Theorem $1.3, d h_{x}(G)=n-1$ for the cut vertex $x$ and $d h_{y}(G)=n-2$ for any end vertex $y$ in $G$, which is a contradiction to the hypothesis. Hence $G=K_{3}$.

Theorem 2.3. Let $G$ be a connected graph of order $n \geq 2$. Then $G=K_{1, n-1}$ if and only if the vertex set $V(G)$ can be partitioned into two sets $V_{1}$ and $V_{2}$ such that $d h_{x}(G)=n-1$ for $x \in V_{1}$ and $d h_{y}(G)=n-2$ for $y \in V_{2}$.

Proof. Suppose that $G=K_{1, n-1}$. Then $d h_{x}(G)=n-1$ for the cut vertex $x$ in $G$ and $d h_{y}(G)=n-2$ for any end vertex $y$ in $G$. Conversely, suppose that the vertex set $V(G)$ can be partitioned into two sets $V_{1}$ and $V_{2}$ such that $d h_{x}(G)=n-1$ for $x \in V_{1}$; and we have $d h_{y}(G)=n-2$ for $y \in V_{2}$. Then by Theorem 1.4, $e_{D}(x)=1$ for each $x \in V_{1}$ and $e_{D}(y)=1$ or $e_{D}(y)=2$ for each $y \in V_{2}$. It follows from Theorem 2.1 that $e_{D}(y)=2$ for some $y \in V_{2}$. Hence $\operatorname{rad}_{D}(G)=1$ and $\operatorname{diam}_{D}(G)=2$. Thus $G=K_{1, n-1}$.

Theorem 2.4. Let $G$ be a connected graph of order $n \geq 5$. Then $G$ is a double star or $G=K_{1, n-1}+e$ if and only if the vertex set $V(G)$ can be partitioned into two sets $V_{1}$ and $V_{2}$ such that $d h_{x}(G)=n-2$ for $x \in V_{1}$ and $d h_{y}(G)=n-3$ for $y \in V_{2}$.

Proof. Suppose that $G$ is a double star or $G=K_{1, n-1}+e$. Then it follows from Theorem 1.3 that $d h_{x}(G)=n-2$ or $d h_{x}(G)=n-3$ according to whether $x$ is a cut vertex of $G$ or not. Conversely, suppose that $d h_{x}(G)=n-2$ for $x \in V_{1}$ and $d h_{x}(G)=n-3$ for $x \in V_{2}$. Then by Theorem 1.4, $e_{D}(x) \leq 3$ for every $x$ and so $\operatorname{diam}_{D}(G) \leq 3$. It follows from Theorem 2.1 that $G \neq K_{2}$ and so $\operatorname{diam}_{D}(G) \geq 2$. If $\operatorname{diam}_{D}(G)=2$, then $G$ is the star $K_{1, n-1}$ and by Theorem $2.3, d h_{x}(G)=n-1$ or $d h_{x}(G)=n-2$ for every vertex $x$. This is a contradiction to the hypothesis. Now, suppose that $\operatorname{diam}_{D}(G)=3$. If $G$ is a tree, then $G$ is a double star. If $G$ is not a tree, then it is clear that $3 \leq \operatorname{cir}(G) \leq 4$, where $\operatorname{cir}(G)$ denotes the length of a longest cycle in $G$. We prove that $\operatorname{cir}(G)=3$. Suppose that $\operatorname{cir}(G)=4$. Let $C_{4}: v_{1}, v_{2}, v_{3}, v_{4}, v_{1}$ be a 4 -cycle in $G$. Since $n \geq 5$ and $G$ is connected, there is a vertex $x$ not on $C_{4}$ such that $x$ is adjacent to some vertex say, $v_{1}$ of $G$. Then $x, v_{1}, v_{2}, v_{4}, v_{4}$ is a path of length 4 in $G$ and so $\operatorname{diam}_{D}(G) \geq 4$, which 
is a contradiction. Thus $\operatorname{cir}(G)=3$. Also, if $G$ contains two or more cycles, then it follows that $\operatorname{diam}_{D}(G) \geq 4$. Hence $G$ contains a unique triangle, say $C_{3}: v_{1}, v_{2}, v_{3}, v_{1}$. Since $n \geq 5$, at least one vertex of $C_{3}$ has degree at least 3 . If there are two or more vertices of $C_{3}$ having degree at least 3 , then $\operatorname{diam}_{D}(G) \geq 4$, which is a contradiction. Thus exactly one vertex of $C_{3}$ has degree at least 3 and it follows that $G=K_{1, n-1}+e$. This completes the proof.

\section{Detour and Vertex Detour Hull Numbers and Addition of A Pendant Edge}

In this section we discuss how the detour hull number and the vertex detour hull number of a connected graph are affected by adding a pendant edge to $G$. Let $G^{\prime}$ be a graph obtained from a connected graph $G$ by adding a pendant edge $u v$, where $u$ is not a vertex of $G$ and $v$ is a vertex of $G$.

Theorem 3.1. If $G^{\prime}$ is a graph obtained from a connected graph $G$ by adding a pendant edge uv at a vertex $v$ of $G$, then $d_{h}(G) \leq d_{h}\left(G^{\prime}\right) \leq d_{h}(G)+1$.

Proof. Let $S$ be a minimum detour hull set of $G$ and let $S^{\prime}=S \cup\{u\}$. We show that $S^{\prime}$ is a detour hull set of $G^{\prime}$. Let $x \in V\left(G^{\prime}\right)$. If $x=u$, then $x \in S^{\prime}$. So, assume that $x \in V(G)$. Then $x \in I_{D}^{k}[S]_{G}$ for some $k \geq 0$. Since $I_{D}^{n}[S]_{G}=I_{D}^{n}[S]_{G^{\prime}}$ for all $n \geq 0$, we have $x \in I_{D}^{k}[S]_{G^{\prime}}$. Also, since $S \subseteq S^{\prime}$, we see that $I_{D}^{n}[S]_{G^{\prime}} \subseteq I_{D}^{n}\left[S^{\prime}\right]_{G^{\prime}}$ for all $n \geq 0$. Hence $x \in I_{D}^{k}\left[S^{\prime}\right]_{G^{\prime}}$. This implies that $S^{\prime}$ is a detour hull set of $G^{\prime}$ so that $d_{h}\left(G^{\prime}\right) \leq\left|S^{\prime}\right|=|S|+1=d_{h}(G)+1$. For the lower bound, let $S^{\prime}$ be a minimum detour hull set of $G^{\prime}$. Then by Theorem 1.1, $u \in S^{\prime}$ and $v \notin S^{\prime}$. Let $S=\left(S^{\prime}-\{u\}\right) \cup\{v\}$. We prove that $S$ is a detour hull set of $G$. For this, first we claim that $I_{D}^{k}\left[S^{\prime}\right]_{G^{\prime}}-\{u\} \subseteq I_{D}^{k}[S]_{G}$ for all $k \geq 0$. We use induction on $k$. Since $S^{\prime}-\{u\} \subseteq S$, the result is true for $k=0$. Let $k=1$ and let $x \in I_{D}\left[S^{\prime}\right]_{G^{\prime}}-\{u\}$. Then $x \neq u$. If $x \in S^{\prime}$, then $x \in S \subseteq I_{D}[S]_{G}$. If $x \notin S^{\prime}$, then there exist $y, z \in S^{\prime}$ such that $x \in I_{D}[y, z]_{G^{\prime}}$ with $x \neq y, z$. If $y \neq u$ and $z \neq u$, then $y, z \in S$ and so $I_{D}[y, z]_{G}=I_{D}[y, z]_{G^{\prime}}$. Thus $x \in I_{D}[S]_{G}$. Now, let $y=u$ or $z=u$, say $z=u$. Since $v$ is a cut vertex of $G^{\prime}$, it follows that $x \in I_{D}[y, v]_{G^{\prime}}=I_{D}[y, v]_{G}$ and hence $x \in I_{D}[S]_{G}$. Assume that the result is true for $k=l$. Then $I_{D}^{l}\left[S^{\prime}\right]_{G^{\prime}}-\{u\} \subseteq I_{D}^{l}[S]_{G}$. Now, let $x \in I_{D}^{l+1}\left[S^{\prime}\right]_{G^{\prime}}-\{u\}$. If $x \in I_{D}^{l}\left[S^{\prime}\right]_{G^{\prime}}$, then by induction hypothesis, we have $x \in I_{D}^{l}[S]_{G} \subseteq I_{D}^{l+1}[S]_{G}$. If $x \notin I_{D}^{l}\left[S^{\prime}\right]_{G^{\prime}}$, then there exist $y, z \in I_{D}^{l}\left[S^{\prime}\right]_{G^{\prime}}$ such that $x \in I_{D}[y, z]_{G^{\prime}}$ with $x \neq y, z$. If $y \neq u$ and $z \neq u$, then it follows from induction hypothesis that $y, z \in I_{D}^{l}[S]_{G}$. Also, since $I_{D}[y, z]_{G^{\prime}}=I_{D}[y, z]_{G}$, we have $x \in I_{D}^{l+1}[S]_{G}$. Let $y=u$ or $z=u$, say $z=u$. Then $y \neq u$ and so by induction hypothesis, $y \in I_{D}^{l}[S]_{G}$. Since $v$ is a cut vertex of $G^{\prime}$, it follows that $x \in I_{D}[y, v]_{G^{\prime}}=I_{D}[y, v]_{G}$. Also, since $v \in S \subseteq I_{D}^{l}[S]_{G}$, it follows that $x \in I_{D}^{l+1}[S]_{G}$. Hence the proof of the claim is complete by induction. Now, since $S^{\prime}$ is a minimum detour hull set of $G^{\prime}$, there is an integer $r \geq 0$ such that $I_{D}^{r}\left[S^{\prime}\right]_{G^{\prime}}=V\left(G^{\prime}\right)$ and it follows from the above claim that $I_{D}^{r}[S]_{G}=V(G)$. Thus $S$ is a detour hull set of $G$ so that $d_{h}(G) \leq|S|=\left|S^{\prime}\right|=d_{h}\left(G^{\prime}\right)$. This completes the proof. 
Remark 3.2. The bounds for $d_{h}\left(G^{\prime}\right)$ in Theorem 3.1 are sharp. Let $G^{\prime}$ be the graph obtained from the graph $G$ in Figure 3, by adding a pendant edge at one of its end vertices. Then $d_{h}\left(G^{\prime}\right)=d_{h}(G)=2$. If $G^{\prime}$ is obtained from $G$ by adding a pendant edge at one of its cut vertices, then $d_{h}\left(G^{\prime}\right)=d_{h}(G)+1$.

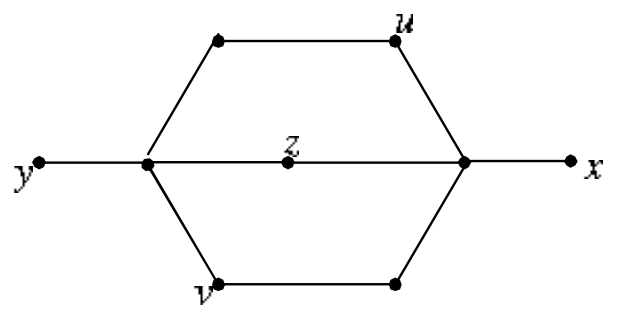

FiguRE 3. Graph $G$ with $d_{h}\left(G^{\prime}\right)=d_{h}(G)+1$

Theorem 3.3. Let $G^{\prime}$ be a graph obtained from a connected graph $G$ by adding a pendant edge uv at a vertex $v$ of $G$. Then $d_{h}(G)=d_{h}\left(G^{\prime}\right)$ if and only if $v$ is a vertex of some minimum detour hull set of $G$.

Proof. First, assume that there is a minimum detour hull set $S$ of $G$ such that $v \in S$. Let $S^{\prime}=(S-\{v\}) \cup\{u\}$. Then $\left|S^{\prime}\right|=|S|$. We show that $S^{\prime}$ is a detour hull set of $G^{\prime}$. First, we claim that $I_{D}^{k}[S]_{G} \subseteq I_{D}^{k+1}\left[S^{\prime}\right]_{G^{\prime}}$ for all $k \geq 0$. We prove this by using induction on $k$. Let $k=0$. Let $x \in S$. If $x \neq v$, then $x \in S^{\prime} \subseteq I_{D}\left[S^{\prime}\right]_{G^{\prime}}$. If $x=v$, then $x \in I_{D}[y, u]_{G^{\prime}} \subseteq I_{D}\left[S^{\prime}\right]_{G^{\prime}}$, where $y \in S$ such that $y \neq v$. Thus $S \subseteq I_{D}\left[S^{\prime}\right]_{G^{\prime}}$. Assume the result for $k=l$. Then $I_{D}^{l}[S]_{G} \subseteq I_{D}^{l+1}\left[S^{\prime}\right]_{G^{\prime}}$. Let $x \in I_{D}^{l+1}[S]_{G}$. If $x \in I_{D}^{l}[S]_{G}$, then by induction hypothesis, $x \in I_{D}^{l+1}\left[S^{\prime}\right]_{G^{\prime}} \subseteq I_{D}^{l+2}\left[S^{\prime}\right]_{G^{\prime}}$. If $x \notin I_{D}^{l}[S]_{G}$, then there exist $y, z \in I_{D}^{l}[S]_{G}$ such that $x \in I_{D}[y, z]_{G}=I_{D}[y, z]_{G^{\prime}}$. By induction hypothesis, we have $y, z \in I_{D}^{l+1}\left[S^{\prime}\right]_{G^{\prime}}$ and so $x \in I_{D}^{l+2}\left[S^{\prime}\right]_{G^{\prime}}$. Hence by induction $I_{D}^{k}[S]_{G} \subseteq I_{D}^{k+1}\left[S^{\prime}\right]_{G^{\prime}}$ for all $k \geq 0$. Now, since $S$ is a detour hull set of $G$, there exists an integer $r \geq 0$ such that $I_{D}^{r}[S]_{G}=V(G)$ and it follows from the above claim that $I_{D}^{r+1}\left[S^{\prime}\right]_{G^{\prime}}=V\left(G^{\prime}\right)$. Thus $S^{\prime}$ is a detour hull set of $G$ so that $d_{h}\left(G^{\prime}\right) \leq\left|S^{\prime}\right|=|S|=d_{h}(G)$. The other inequality follows from Theorem 3.1.

Conversely, let $d_{h}(G)=d_{h}\left(G^{\prime}\right)$. Let $S^{\prime}$ be a minimum detour hull set of $G^{\prime}$. Then by Theorem 1.3, $u \in S^{\prime}$ and $v \notin S^{\prime}$. Let $S=\left(S^{\prime}-\{u\}\right) \cup\{v\}$. Then, as in the proof of Theorem 3.1, we can prove that $S$ is a detour hull set of $G$. Since $|S|=\left|S^{\prime}\right|=d_{h}\left(G^{\prime}\right)=d_{h}(G)$, we see that $S$ is a minimum detour hull set of $G$ and $v \in S$. This completes the proof.

Theorem 3.4. Let $G$ be a connected graph and let $x$ be any vertex in $G$. If $G^{\prime}$ is a graph obtained from $G$ by adding a pendant edge $x u$, then $d h_{x}\left(G^{\prime}\right)=d h_{x}(G)+1$.

Proof. Let $S$ be a minimum $x$-detour hull set of $G$ and let $S^{\prime}=S \cup\{u\}$. Then, as in Theorem 3.1, it is straight forward to verify that $I_{D}^{n}[S]_{G}^{x} \subseteq I_{D}^{n}\left[S^{\prime}\right]_{G^{\prime}}^{x}$ for all $n \geq 0$. Since $S$ is an $x$-detour hull set of $G$, there is an integer $r \geq 0$ such that 
$I_{D}^{r}[S]_{G}^{x}=V(G)-\{x\}$ and it is clear that $I_{D}^{r}\left[S^{\prime}\right]_{G^{\prime}}^{x}=V\left(G^{\prime}\right)-\{x\}$. Hence $S^{\prime}$ is an $x$-detour hull set of $G^{\prime}$ so that $d h_{x}\left(G^{\prime}\right) \leq\left|S^{\prime}\right|=d h_{x}(G)+1$. Now, suppose that $d h_{x}\left(G^{\prime}\right)<d h_{x}(G)+1$. Let $S^{\prime}$ be a minimum $x$-detour hull set of $G^{\prime}$. Then, by Theorem 1.3, $u \in S^{\prime}$. Let $S=S^{\prime}-\{u\}$. Then, as in Theorem 3.1, it is straight forward to prove that $I_{D}^{n}\left[S^{\prime}\right]_{G^{\prime}}^{x}-\{u\} \subseteq I_{D}^{n}[S]_{G}^{x}$ for all $n \geq 0$. Since $S^{\prime}$ is an $x$-detour hull set of $G^{\prime}$, there is an integer $r \geq 0$ such that $I_{D}^{r}\left[S^{\prime}\right]_{G^{\prime}}^{x}=V\left(G^{\prime}\right)-\{x\}$. Hence $I_{D}^{r}[S]_{G}^{x}=V(G)-\{x\}$. Thus $S$ is an $x$-detour hull set of $G$ so that $d h_{x}(G) \leq|S|=$ $d h_{x}\left(G^{\prime}\right)-1$, which is a contradiction to $d h_{x}\left(G^{\prime}\right)<d h_{x}(G)+1$. Hence the result follows.

Theorem 3.5. Let $G^{\prime}$ be a graph obtained from a connected graph $G$ by adding a pendant edge uv at a vertex $v$ of $G$. Then $d h_{u}\left(G^{\prime}\right)=d h_{v}(G)$.

Proof. Let $S$ be a minimum $v$-detour hull set of $G$. Then $v \notin S$. As in Theorem 3.1, it is straight forward to prove that $I_{D}^{n}[S]_{G}^{v} \subseteq I_{D}^{n}[S]_{G^{\prime}}^{u}$ for all $n \geq 0$. Since $S$ is a $v$ detour hull set of $G$, there is an integer $r \geq 0$ such that $I_{D}^{r}[S]_{G}^{v}=V(G)-\{v\}$. Now, since $v \in I_{D}[z]_{G}^{u}$ for any $z \in S$, it follows that $I_{D}^{r}[S]_{G}^{u}=V\left(G^{\prime}\right)-\{u\}$. Hence $S$ is a $u$ detour hull set of $G^{\prime}$ so that $d h_{u}\left(G^{\prime}\right) \leq|S|=d h_{v}(G)$. For the other inequality, let $T$ be a minimum $u$-detour hull set of $G^{\prime}$. Then $u \notin T$ and by Theorem 1.3(iii), $v \notin T$. As in Theorem 3.1, it is straight forward to prove that $I_{D}^{n}[T]_{G^{\prime}}^{u}-\{v\} \subseteq I_{D}^{n}[T]_{G}^{v}$ for all $n \geq 0$. Since $T$ is a $u$-detour hull set of $G^{\prime}$, there is an integer $r \geq 0$ such that $I_{D}^{r}[T]_{G^{\prime}}^{u}=V\left(G^{\prime}\right)-\{u\}$. Hence it follows that $I_{D}^{r}[T]_{G}^{v}=V(G)-\{v\}$ and $T$ is a $v$-detour hull set of $G$. Thus $d h_{v}(G) \leq|T|=d h_{u}\left(G^{\prime}\right)$. This completes the proof.

Theorem 3.6. Let $G$ be a connected graph and $x$ any vertex of $G$. Let $G^{\prime}$ be a graph obtained from $G$ by adding a pendant edge uv at a vertex $v \neq x$ of $G$. Then $d h_{x}(G) \leq d h_{x}\left(G^{\prime}\right) \leq d h_{x}(G)+1$.

Proof. The proof is similar to Theorem 3.1.

Theorem 3.7. Let $G$ be a connected graph and $x$ any vertex of $G$. Let $G^{\prime}$ be a graph obtained from $G$ by adding a pendant edge uv at a vertex $v \neq x$ of $G$. Then $d h_{x}(G)=d h_{x}\left(G^{\prime}\right)$ if and only if $v$ belongs to some minimum $x$-detour hull set of $G$.

Proof. The proof is similar to Theorem 3.3. 


\section{REFERENCES}

[1] Buckley, F., and Harary, F., Distance in Graphs, Addison-Wesley, Reading MA, 1990.

[2] Chartrand, G., Escuadro, H., and Zhang, P., "Detour distance in graphs", J. Combin. Math. Combin. Comput., 53 (2005), 75-94.

[3] Chartrand, G., Johns, G.L., and Zhang P., "Detour number of a graph", Util. Math., 64 (2003), 97-113.

[4] Chartrand, G., Johns, G.L., and Zhang P., "On the detour number and geodetic number of a graph", Ars Combinatoria, 72 (2004), 3-15.

[5] Chartrand, G., Nebesky, L., and Zhang, P., "A survey of Hamilton colorings of graphs", Preprint.

[6] Chartrand, G., and Zhang, P., Introduction to Graph Theory, Tata McGraw- Hill Edition, New Delhi, 2006.

[7] Hale, W., "Frequency Assignment; Theory and Applications", Proc. IEEE, 68 (1980), 14971514.

[8] Santhakumaran, A.P., and Athisayanathan, S., "Connected detour number of a graph", J. Combin. Math. Combin. Comput., 69 (2009), 205-218.

[9] Santhakumaran, A.P., and Titus, P., "The vertex detour number of a graph", $A K C E J$. Graphs. Combin., 4:1 (2007), 99-112.

[10] Santhakumaran, A.P., and Ullas Chandran, S.V., "The vertex detour hull number of a graph", Discuss. Math. Graph Theory, 32 (2012), 319 -328.

[11] Santhakumaran, A.P., and Ullas Chandran, S.V., "The detour hull number of a graph", Algebra \& Discrete Math., 14:2 (2012), 307 - 322. 